\title{
Mechanical Behavior and Characterization of Stern-shaft Mechanical Sealing Device
}

\author{
Yongjin Lu ${ }^{a}{ }^{*}$, Rui Lin \\ China Ship Development and Design Center, Wuhan, 430064, China \\ asoda1998@126.com
}

\begin{abstract}
Keywords: Stern-shaft seal; Spring piece; In-situ compression test; Nanoindentation; Surface morphology
\end{abstract}

Abstract: For the stern-shaft mechanical sealing device, spring pieces and sealing rings are the main study objects of mechanical behavior and characterization. Consequently, the in-situ compression tests for the spring pieces were performed, where special test equipments were applied to ensure the measurement accuracy. It was found that the load-displacement relationships of supporting spring piece, bridging spring piece and bracket spring piece corresponded to the formulas $F=1.02 L^{0.458}$, $F=0.2 L^{1.317}$ and $F=0.165 L^{1.236}$. Furthermore, the nanoindentation test of rotary ring material was carried out, and the average elastic modulus and hardness of tin bronze were $118.79 \mathrm{GPa}$ and 3.96 $\mathrm{GPa}$, respectively. Surface morphology test for the seal face was conducted. It is revealed that phenolic resin has a stronger wear resistance compared with tin bronze, because the metal particles embedded in the phenolic resin cause the wear of tin bronze. Hence the characterization methods can accurately predict the mechanical properties of the device and meet the requirements of ship design.

\section{Introduction}

At present, as one of the important auxiliary equipments for propeller shaft system, stern-shaft mechanical sealing device is installed on the stern shaft penetrated through the hull, which achieve the ship watertight function. With the development of emerging technologies, mechanical seal technology has been used widely for the domestic market, which involves one pair of sliding face perpendicular to the shaft axis and spring assembly, maintaining a certain pressure to ensure the long-term effective operation of mechanical seal.

Stern-shaft mechanical sealing device generally consists of hermetic sealing seat, hermetic sealing ring, bracket, spring assembly, stationary ring, rotary ring, etc. The device structure can be simplified as an effective sealing pair composed of the rotary ring and the stationary ring bearing spring compression force. Thus, spring assembly and mechanical seal face are important parts of the stern-shaft mechanical sealing device, which determines the performance and service life of the whole device. However, there are no open publications describing the experimental study on the sealing materials. In this study, means and methods used for characterizing the mechanical behavior of the stern-shaft mechanical sealing device were elaborated comprehensively, and the compression tests for the typical spring pieces were established. Furthermore, the experimental tests and the surface topography studies on the sealing material were carried out.

\section{In-situ compression tests for the spring pieces}

As an elastic compensation component for the sealing device, the spring assembly is composed of supporting spring pieces, bridging spring pieces, bracket spring pieces and other pieces. During the actual work process, the spring assembly is in compression, and there exists a force acting on the stationary ring to make the sealing fit closely, which ensure the sealing device no leakage. Simultaneously, supporting spring pieces, bridging spring pieces, bracket spring pieces, as the shell structure, are made of Monel alloy. Different geometrical structures may lead to different deformation mechanisms, which might affect the mechanical properties of spring assembly. Juxi $\mathrm{Hu}^{[1,2]}$ constructed numerical models for the spring pieces and spring assembly using the finite 
element method, and investigated the influence of root circle radius and shell thickness on mechanical properties. However, due to the lack of the experimental results, it is difficult to provide guidances for the spring assembly on theory analysis and structure design.

In general, the mechanical properties of metal sheets are usually obtained by conventional tensile test. Owing to ensuring the consistency and the versatility of mechanical test results, the sheets are processed into tensile specimens by the national standard GB/T 228-2002. Through the tensile test, the relationship between force and displacement is acquired, and need be converted into the true stress-strain curve, then calculate the material mechanical parameters, such as elastic modulus, tensile strength, strain hardening exponent and so on.

For the spring assembly, its mechanical performance is not only related to the material properties, but also sensitive to the other factors, including structure shape and geometrical dimensions. Hence its compression performance is seriously different from tensile property. For the sake of measurement accuracy, it is necessary to design the special tools for in-situ compression tests of the spring pieces. As illustrated in Fig. 1, spring piece is clamped at one end to fix constraint, and the vertical load is applied to the other free end of the piece toward fixed end by the test machine, then the compression load and displacement data can be obtained online, subsequently the relationship between force and displacement is calculated. Taking a certain type of stern-shaft mechanical sealing device as an example, the expansion length of supporting spring piece, bridging spring piece and bracket spring piece was $325 \mathrm{~mm}, 346 \mathrm{~mm}, 436 \mathrm{~mm}$, respectively, and the specimens were Monel alloy sheets about $0.6 \mathrm{~mm}$ in thickness and $25 \mathrm{~mm}$ in width. In order to ensure the accuracy and reliability of test data, each spring piece for compression test was performed three times, and the test results were averaged. As shown in Fig. 2, it is revealed that the load-displacement relationship of supporting spring piece appear power function distribution and the exponent is less than 1, corresponding to the formula $F=1.02 L^{0.458}$. Moreover, the load-displacement relationships of bridging spring piece and bracket spring piece corresponded to the formulas $F=0.2 L^{1.317}$ and $F=0.165 L^{1.236}$, respectively, and the index of the formulas is significantly larger than 1 . Through reasonable design, it can be achieved that the load-displacement relationship of the spring assembly appears approximately linear.

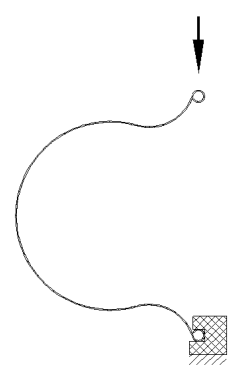

a) Supporting spring piece

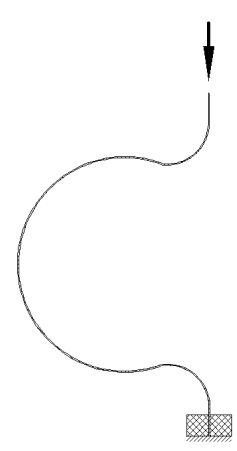

b) Bridging spring piece

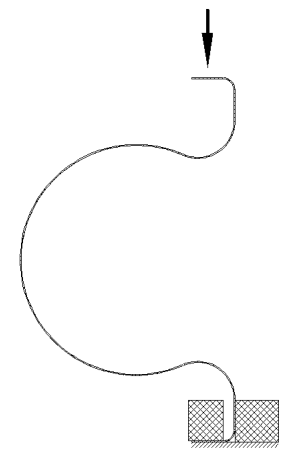

c) Bracket spring piece

Fig.1 In-situ compression tests for different spring pieces 


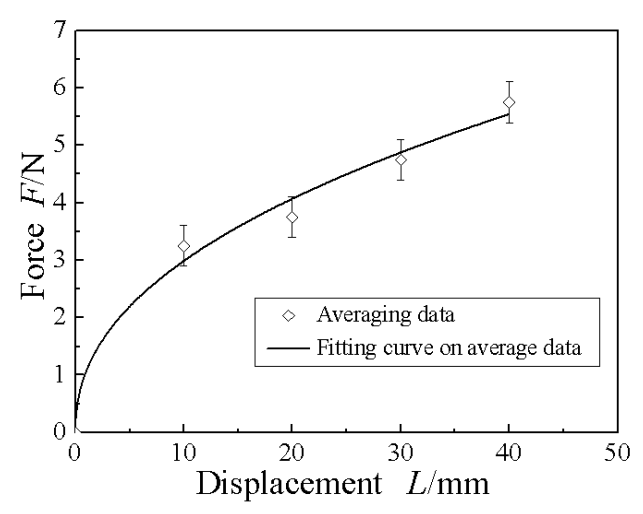

a) Supporting spring piece

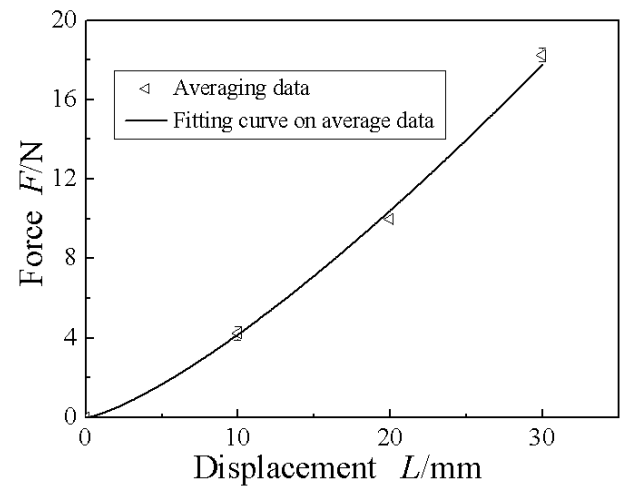

b) Bridging spring piece

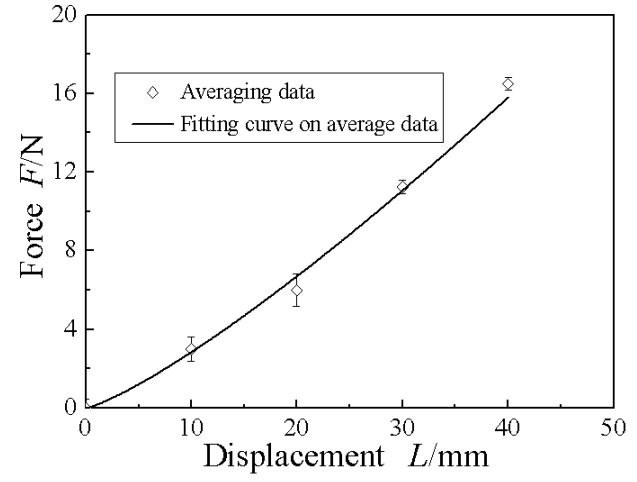

c) Bracket spring piece

Fig.2 Load-displacement curves for typical spring pieces

\section{Nanoindentation test for rotary ring}

According to the requirements and conditions of the stern-shaft mechanical sealing device, the objective of rotary ring material should have good wear resistance, strong corrosion resistance, and not easy to produce scuffing. Therefore, the tin bronze material is usually processed by centrifugal casting in the forming process. As one of the most important indicators in the mechanical properties of metallic materials, hardness reflects elasticity, plasticity, strength, deformation enhancement rate and antifriction property of material, when a compressive force is applied to the test material. Hence the microhardness test can be regarded as a significant method to study the microcosmic properties of materials, which has been applied widely in tribology research ${ }^{[3-5]}$.

In this research, the composition of the commercial ZCuAl8Mn13Fe3Ni2 tin bronze alloy used for testing was $73.3 \mathrm{Cu}-7.95 \mathrm{Al}-13.11 \mathrm{Mn}$ (wt.\%), and the final products of $\varnothing 20 \mathrm{~mm} \times 6 \mathrm{~mm}$ were prepared. The elastic modulus and hardness of rotary ring material were measured by Triboindenter nanomechanical testing system, and the main geometry parameters involved in the test can be found in the following list: Burke's diamond indenter; angle of plane: $142.3^{\circ}$; indenter tip radius: $100 \mathrm{~nm}$; loading rate: $0.05 \mathrm{~s}^{-1}$; indentation depth: $2 \mathrm{um}$. For comparison, indentation locations were selected on the material surface at intervals of $45^{\circ}$. The load-displacement curves of tin bronze were obtained respectively, then the elastic modulus was calculated by the slope of the unloading curve, subsequently the hardness value was obtained by the maximum load and the residual deformation area of the indentation. The results showed that the average elastic modulus and hardness of tin bronze were $118.79 \mathrm{GPa}$ and $3.96 \mathrm{GPa}$, respectively, the average variance of elastic modulus and hardness were $0.849 \mathrm{GPa}$ and $0.015 \mathrm{GPa}$, as shown in Fig. 3. Therefore, the mechanical properties of the tin bronze were very stable, and there was no obvious fluctuation phenomenon.

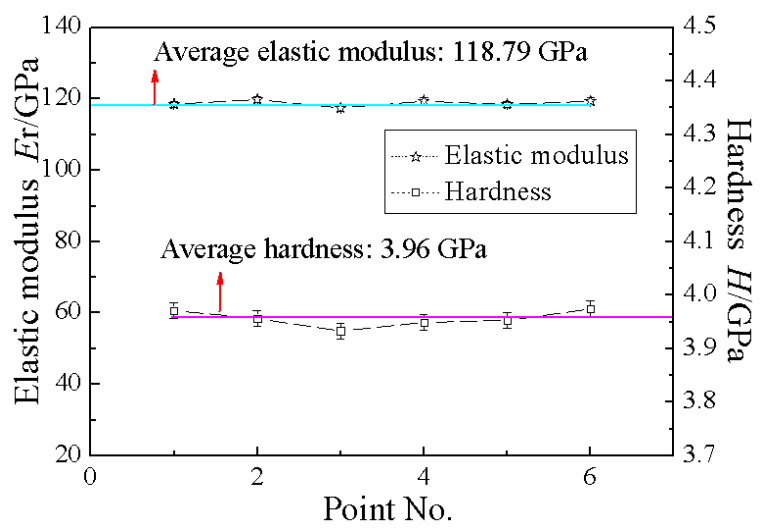

Fig.3 Nanoindentation test data of rotary ring material 


\section{Surface morphology test for the seal face}

In order to obtain the high precision surface quality, the seal faces of stationary and rotary ring are usually manufactured by the fine machining. But there is still uneven and a certain surface roughness for the seal faces on a micro scale level, so it reveals that partial contact contributes to the gap with each other on the seal. With the relative rotation between the rings, the liquid flows from one gap to another, and has been to the seal edge and thus leakage. According to the literature[6,7], it showed that the surface roughness and the end face gap are in the same order of magnitude. Consequently, the surface roughness is the most important surface geometrical feature of the sealing performance, and can be expressed by extracting the contour surface of a certain region on the surface.

Relying on the shafting attachment performance test platform in china ship development and design center, the test was measured to analyze the surface morphologies. During the test, the diameter of the stern shaft used was $420 \mathrm{~mm}$, the rotary and stationary ring materials were tin bronze and phenolic resin material respectively, wear test lasted 300 hours. The pressure applied on the stationary ring side was $0.2 \mathrm{MPa}$, the medium pressure was $0.04 \mathrm{MPa}$, rotating speed of the dynamic ring was $220 \mathrm{r} / \mathrm{min}$. Utilizing a CETR-UMT-1 Tribometer, the surface morphologies of the typical 0.5 $\mathrm{mm} \times 0.5 \mathrm{~mm}$ area were selected from the surface of the stationary and rotary ring before and after wear. In addition, the scan accuracy was as follows: $10 \times 10-500 \times 500 \mathrm{um}^{2}$, horizontal resolution was $0.1 \mathrm{um}$, vertical resolution was $0.1 \mathrm{~nm}$, step height measurement repeatability was $0.8 \mathrm{~nm}$. Comparing the weighted average of the test results, the calculation formula is as follows:

$$
R_{\mathrm{a}}=\frac{1}{l} \int_{0}^{l}|z(x)| \mathrm{d} x=\frac{1}{m} \sum_{i=1}^{m}\left|z_{i}\right|
$$

In the formula, $z(x)$ is each point contour height; $L$ is measurement length; $m$ is measurement times; $z_{i}$ is contour height of each measuring point.

Surface morphology changes of different sealing materials before and after wear are shown in Fig. 4, average surface roughness of the stationary ring decreased from 0.512 um to 0.314 um, while average surface roughness of the rotary ring increased from $0.337 \mathrm{um}$ to $0.632 \mathrm{um}$. This indicates that the phenolic resin has a stronger wear resistance in the sealing material combination of soft and hard materials. A surface morphology of the stationary ring after wear is presented in Fig. 5, it can be found there are some convex peaks in the surface after wear. In the wear process, the stationary ring surface is embedded in fine tin bronze particles. Instead, the fine metal particles caused a certain wear to the tin bronze body, which make the surface roughness increase. At the same time, according to the seal structure, the rough surface of stern-shaft mechanical sealing device meet the radial parallel face seal model. It can be proved that the running gap is proportional to the total surface roughness of the stationary and rotary ring. As seen in Fig.4, the total surface roughness increases from 0.849 um to $0.946 \mathrm{um}$, the test result indicates that the entire wear test increased the gap, which was demonstrated by the leakage increasing from $2.23 \mathrm{~L} / \mathrm{h}$ to $2.37 \mathrm{~L} / \mathrm{h}$. Therefore, for the operation and management of the device, the service life and maintenance time of the seal are qualitatively judged by measuring the surface roughness of the rings during the voyage.

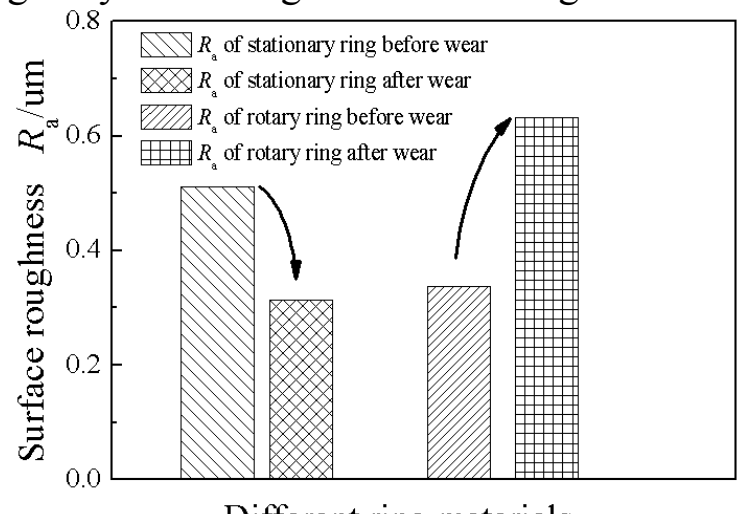

Different ring materials

Fig.4 Surface microtopography changes for different sealing materials in wear process 


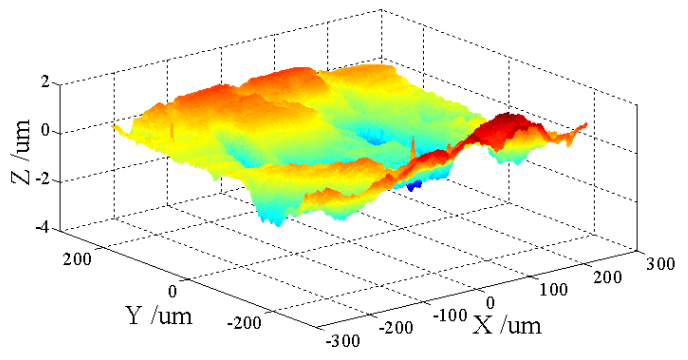

a) Before wear

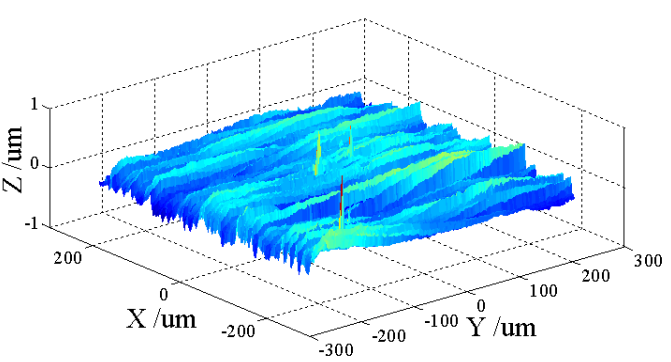

b) After wear

Fig.5 Surface microtopographies for stationary ring materials before and after wear

\section{Conclusions}

The experimental methods used for characterizing the device are elaborated comprehensively from three aspects: in-situ compression tests for the spring pieces, nanoindentation test for rotary ring, surface morphology test for the seal face, and the tests obtained the following conclusions:

1) Based on the special working equipment, in-situ compression tests for three kinds of spring pieces including supporting spring pieces, bridging spring pieces, bracket spring pieces, were constructed, and revealed the typical mechanical spring deformation behaviors. It was found that the exponent of load-displacement function for the supporting spring piece is less than 1, and the relationship between the force and displacement of bridging and bracket spring piece appears power function distribution with exponent greater than 1.

2) Taking the rotary ring as the research object, tin bronze was performed in nanoindentation test, and the results showed that the average elastic modulus and hardness of tin bronze were $118.79 \mathrm{GPa}$ and $3.96 \mathrm{GPa}$, respectively;

3 ) The surface morphology test for the seal face was performed before and after wear, where the device lasted 300 hours wear test. It was found that phenolic resin has a stronger wear resistance compared with tin bronze, because the metal particles embedded in the phenolic resin cause the wear of tin bronze. Experimental results verified the phenomenon that the surface gap is proportional to the total surface roughness of the sealing surface.

\section{Acknowledgements}

The authors wish to express gratitude to Dept. of Mechanical Engineering Tsinghua University for providing the research facilities used to undertake this work.

\section{References}

[1] Juxi Hu, Long Chang, Haifeng Yang, Xiaojun Wang. Ship engineering, 2010, 32(2): 82-84.

[2] Juxi Hu, Long Chang, Haifeng Yang, Xiaojun Wang. Ship engineering, 2010, 32(6): 21-23, 47.

[3] Dewei Han. Metal hardness testing manual. Changsha: Central South University press, 2003:18-23.

[4] Jiajun Liu. Material wear principle and its wear resistance. Beijing: Tsinghua University press. 1993:1.

[5] Ming Li, Shizhu Wen. China Mechanical Engineering, 2002, 13(16): 1437-1439.

[6] Shugui Zhang, Yongquan Gu. Journal of Petroleum University, 1992, 16( 2) : 48-53.

[7] Yongquan Gu. Mechanical end seal. Dongying: Oil university press, 1994:3-7.

[8] N Brunetiere, B Modolo. International Journal of Thermal Sciences, 2009, 48(4):781-794. 
